\title{
Viscous behaviour of glass-forming liquids: a thermodynamic approach
}

\author{
R K MISHRA and K S DUBEY* \\ Department of Applied Physics, Institute of Technology, Banaras Hindu University, \\ Varanasi 221005 , India
}

MS received 2 May 1996

\begin{abstract}
The temperature dependence of the viscosity of the undercooled melts exhibits an important role in the study of nucleation, crystal growth and the glass-forming ability of materials. Several attempts have been made to study the viscous behaviour of the glassforming melts and these investigations are mainly based on free volume theory as well as on the configurational entropy model. In the present investigation, an attempt has been made to correlate the thermodynamic parameters with the viscosity of the glass-forming melts and to study the temperature dependence of the viscosity of undercooled liquids on the basis of the free volume theory as well as on the basis of the configurational entropy model of Adam and Gibbs. The entire study is confined on the expression for thermodynamic parameters reported by the authors recently. The expression obtained has been successfully applied to study the temperature dependence of the viscosity of the metallic, molecular and oxide glass-forming liquids.
\end{abstract}

Keywords. Viscosity; free volume; configurational entropy; Kauzmann temperature; thermodynamic parameters.

\section{Introduction}

The viscosity $(\eta)$ and the Gibbs free energy difference $(\Delta G)$ between the undercooled liquid and the corresponding equilibrium solid are key factors in metastable solidification of undercooled melts. The viscosity $(\eta)$ is also known to be one of the most sensitive variable controlling the glass-forming ability of materials and it is found that there is a large increase in $\eta$ with progressive undercooling (Uhlmann 1972; Davies 1976). The metastability of liquids precludes the experimental determination of $\eta$ of an amorphous alloy between the glass transition temperature $\left(T_{\mathrm{g}}\right)$ and the equilibrium melting temperature $\left(T_{\mathrm{m}}\right)$ due to inability to prevent crystallization. $\eta$ of the glassforming melts can be measured at low temperatures near $T_{\mathrm{g}}$ and at high temperatures above $T_{\mathrm{m}}$. Due to these experimental difficulties, theoretical approaches or crude approximations are commonly used to estimate the viscosity at intermediate temperatures by connecting low and high temperature data of viscosity by simple equations. However, where $T_{\mathrm{g}}$ is well separated from the crystallization temperature $\left(T_{\mathrm{c}}\right)$, it is possible to measure $\eta$ experimentally.

Several expressions (Vogel 1921; Raman 1923; Doolittle 1951; Macedo and Litovitz 1965; Cohen and Grest 1979) have been proposed for the temperature dependence of $\eta$. The simplest expression for $\eta$ is the standard Arrhenius-type equation (Dunn 1926)

$$
\eta=A_{1} \exp (E / R T)
$$

where $E$ is the activation energy for viscous flow, $R$ the gas constant and $A_{1}$ a constant. The above is applicable only in a narrow temperature range for simple liquids and viscosities of associated and network liquids generally do not follow this equation. The 
commonly used expression for describing the temperature dependence of $\eta$ is given by Vogel (1921) and Fulcher (1925) as

$$
\eta=A_{2} \exp \left(\frac{B_{2}}{T-T_{0}}\right)
$$

where $A_{2}$ and $B_{2}$ are constants and $T_{0}$ the characteristic temperature. Often the temperature dependence of viscosity can not easily be explained on the basis of the above stated equation over the entire temperature range of interest with a single set of constants $A_{2}, B_{2}$ and $T_{0}$ (Pok and Turnbull 1972).

There are basically two approaches to obtain an expression for the temperature dependence of $\eta$, namely the free volume theory (Doolittle 1951; Cohen and Turnbull 1959) and the configurational entropy model (Adam and Gibbs 1965). The expressions proposed by Eyring and his co-workers (Glasstone et al 1941; Hirai and Eyring 1958, 1959; Ree et al 1962) and Cohen and Turnbull (1959) are basically based on the diffusion theory while the procedure developed by Adam and Gibbs (1965) is confined on the configurational entropy of undercooled liquids. Recently, the authors (1996) reported an expression for the Gibbs free energy difference $(\Delta G)$ between the undercooled liquid and the corresponding equilibrium solid using the hole theory of liquids (Frankel 1955; Hirai and Eyring 1958, 1959) and the expression obtained has been successfully applied to study the various thermodynamic parameters of metallic, molecular and oxide glass-forming melts. The aim of the present investigation is to study the temperature dependence of the viscosity of the glass-forming melts using expressions for the thermodynamic parameters reported by the authors recently. The viscous behaviour of glass-forming liquids has been studied on the basis of the free volume theory as well as on the basis of the configurational entropy model proposed by Adam and Gibbs (1965). The expression obtained is also found to encompass many empirical relations under appropriate approximations. The expression obtained has been successfully applied to study the temperature dependence of $\eta$ of the metallic $\left(\mathrm{Au}_{0 \cdot 77} \mathrm{Ge}_{0.136} \mathrm{Si}_{0.094}\right)$, oxide $\left(\mathrm{B}_{2} \mathrm{O}_{3}\right)$ and molecular (2-methyl pentane) glass-forming liquids.

\section{Thermodynamic parameters}

Following the earlier work of Dubey and Ramachandrarao (1984) and incorporating the modifications suggested by Sanchez (1974) and Flory (1953), various thermodynamic parameters the Gibbs free energy difference $(\Delta G)$, enthalpy difference $(\Delta H)$ and entropy difference $(\Delta S)$ between the undercooled liquid and solid phases of the glass-forming melts have been studied by the authors (1996) on the basis of the hole theory (Frankel 1955; Hirai and Eyring 1958, 1959) of liquids. According to the earlier report of authors (1996), the specific heat difference $\Delta C_{\mathrm{p}}$ between the undercooled liquid and the equilibrium solid can be expressed as

$$
\Delta C_{\mathrm{p}}=n R\left(\frac{E_{\mathrm{h}}}{R T}\right)^{2} g=\Delta C_{\mathrm{p}}^{\mathrm{m}}\left(\frac{T_{\mathrm{m}}}{T}\right)^{2} e^{-2 \delta \cdot \Delta T / T},
$$

and

$$
g=\exp \left[-\left(\frac{\varepsilon_{\mathrm{h}}+p v_{\mathrm{h}}}{K_{\mathrm{B}} T}+A\right)\right]
$$


with $A=1-1 / n . \Delta T=T_{\mathrm{m}}-T$ represents the degree of undercooling, $E_{\mathrm{h}}$ the hole formation energy per mole, $v_{\mathrm{h}}$ the volume of a hole $n=v_{\mathrm{a}} / v_{\mathrm{h}}$ is a measure of the relative volume of a hole and an atom, $v_{\text {a }}$ the hard core volume per atom or molecule, $\delta=T_{0} / T_{\mathrm{m}}, T_{0}$ the ideal glass transition temperature, $p$ the external pressure and $g$ the volume fraction of holes at temperatures $T . \Delta C_{\mathrm{p}}^{\mathrm{m}}$ is the specific heat difference between the liquid and solid phases at $T=T_{\mathrm{m}} . K_{\mathrm{B}}$ is the Boltzmann constant and $\varepsilon_{\mathrm{h}}$ the energy required for the formation of a hole.

The expressions for $\Delta G, \Delta H$ and $\Delta S$ can be obtained with the aid of basic thermodynamic relations

$$
\begin{aligned}
& \Delta G=\Delta H-T \Delta S, \\
& \Delta H=\Delta H_{\mathrm{m}}-\int_{T}^{T_{\mathrm{m}}} \Delta C_{\mathrm{p}} \mathrm{d} T, \\
& \Delta S=\Delta S_{\mathrm{m}}-\int_{T}^{T_{\mathrm{m}}}\left(\Delta C_{\mathrm{p}} / T\right) \mathrm{d} T,
\end{aligned}
$$

and the expression for $\Delta C_{\mathrm{p}}$ as stated in (3). Consequently one can have

$$
\begin{aligned}
& \Delta G=\Delta S_{\mathrm{m}} \Delta T-\frac{\Delta C_{\mathrm{p}}^{\mathrm{m}}}{4 \delta^{2}}\left[2 \delta \Delta T-T\left(1-e^{-2 \delta . \Delta T / T}\right)\right], \\
& \left.\Delta H=\Delta H_{\mathrm{m}}-\Delta C_{\mathrm{p}}^{\mathrm{m}} \frac{T_{\mathrm{m}}}{2 \delta}\left[1-e^{-2 \delta . \Delta T / T}\right)\right], \\
& \Delta S=\Delta S_{\mathrm{m}}-\frac{\Delta C_{\mathrm{p}}^{\mathrm{m}}}{4 \delta^{2}}\left[(1+2 \delta)-\left(1+2 \delta \frac{T_{\mathrm{m}}}{T}\right)\left(e^{-2 \delta . \Delta T / T}\right)\right],
\end{aligned}
$$

where $\Delta H_{\mathrm{m}}$ and $\Delta S_{\mathrm{m}}$ are enthalpy of fusion and entropy of fusion respectively. As argued by Kauzmann (1948) that a liquid loses its entropy at a faster rate than the solid resulting in the two phases having the equal entropy at some temperature $T_{0}$ well above the absolute zero and below the glass transition temperature $\left(T_{\mathrm{g}}\right)$. The iso-entropy temperature $T_{0}$ is usually referred to as the Kauzmann temperature or the ideal glass transition temperature. Introducing the Kauzmann temperature $T_{0}$ and volume fraction $g$ as stated in (4) the expression for $\Delta H$ stated in (9) takes the form of

$$
\Delta H=\Delta H_{0}+\frac{\Delta C_{\mathrm{p}}^{\mathrm{m}} T_{\mathrm{m}}}{2 \delta g_{\mathrm{m}}}\left(g-g_{0}\right)
$$

where $g_{0}$ and $g_{\mathrm{m}}$ represent volume fractions of holes at temperatures $T_{0}$ and $T_{\mathrm{m}}$ respectively.

As stated earlier, according to Kauzmann $\Delta S$ becomes zero at $T=T_{0}$. In view of it, the expression for $\Delta S$ stated in (10) results as

$$
\Delta S_{\mathrm{m}}=\frac{\Delta C_{\mathrm{p}}^{\mathrm{m}}}{4 \delta^{2}}\left[(1+2 \delta)-3 e^{-2(1-\delta)}\right]
$$

Using the approximations

$$
e^{2 x}-1 \simeq 2 x \frac{3+x}{3-2 x}
$$


and

$$
1-e^{-2 x} \simeq 2 x \frac{3-x}{3+2 x}
$$

and taking the help of (12), expression for $\Delta S$ stated in (10) reduces to

$$
\Delta S=\frac{\Delta C_{\mathrm{p}}^{\mathrm{m}} e^{-2(1-\delta)}}{2 \delta^{2}} \cdot \frac{\left(T-T_{0}\right)\left(3 T-T_{0}\right)}{T^{2}} .
$$

\section{Viscosity of undercooled liquids based on free volume theory}

The free volume model of the viscosity is essentially based on the idea that a certain critical volume must be available to a molecule or an atom before performing a diffusive motion. It was Doolittle (1951) first of all who proposed an empirical relation between viscosity and free volume as

$$
\eta=A \exp \left(B / f_{T}\right)
$$

where $A$ and $B$ are constants and $f_{T}$ the relative free volume fraction at temperature $T$. A theoretical explanation to this relation was given by Cohen and Turnbull (1959) Turnbull and Cohen $(1961,1970)$ as well as by Bueche (1959). The free volume can be defined as unfilled space between atoms or molecules of the material under study. It can also be defined in terms of the thermal expansion and the hole theory of liquids and has been summarized by Bondi (1954). Following the earlier work of Doolittle (1951) and Ramachandrarao et al (1977), the relative free volume $f_{T}$ can be expressed as

$$
f_{T}=\frac{V_{T}-V_{T_{0}}}{V_{T_{0}}}
$$

where $V_{T}$ and $V_{T_{0}}$ are volumes of material at temperatures $T$ and at some reference temperature $T_{0}$. Consequently, in terms of volume fractions of holes, it can also be expressed as

$$
f_{T}=\frac{g-g_{0}}{1-g} .
$$

Utilizing the Doolittle equation as stated in (15) and using (17), one can have

$$
\eta=A_{3} \exp \left(\frac{B_{3}}{g-g_{0}}\right)
$$

Here a term $\left(1-g_{0}\right)$ has been absorbed in the constant $B_{3} . A_{3}$ is also a constant. Use of (11) allows one to express

$$
g-g_{0}=\left(\Delta H-\Delta H_{0}\right) \frac{2 \delta g_{\mathrm{m}}}{\Delta C_{\mathrm{p}}^{\mathrm{m}} T_{\mathrm{m}}} .
$$

Taking the aid of (18) and (19) one can express the viscosity of the glass-forming melts in terms of thermodynamic quantities as

$$
\ln \eta=\ln \eta_{0}+\frac{2 B_{3} \delta g_{\mathrm{m}}}{\Delta C_{\mathrm{p}}^{\mathrm{m}} T_{\mathrm{m}}} \cdot \frac{1}{\Delta H-\Delta H_{0}} \equiv \ln \eta_{0}+\frac{D}{\Delta H-\Delta H_{0}},
$$


which clearly shows the evaluation of $\eta$ with the aid of enthalpy differences $\Delta H$ and $\Delta H_{0}$ at temperatures $T$ and $T_{0}$ respectively. Here $D$ can be taken as a constant. Appropriate substitution of $\Delta H$ and $\Delta H_{0}$ in terms of $\Delta G$ and $\Delta S$ and simplification of (20) yields

$$
\ln \eta=\ln \eta_{0}+\frac{D}{T \Delta S\left[1-\frac{\Delta G_{0}-\Delta G}{T \Delta S}\right]}
$$

where $\Delta G_{0}$ stands for the Gibbs free energy difference at $T=T_{0}$ and $\eta_{0}$ is a constant. Using the approximation stated in (13), it can be shown that

$$
\Delta G_{0}-\Delta G=\frac{T \Delta S\left(1-T_{0} / T\right)}{\left(1-T_{0} / 3 T\right)\left(1+2 T_{0} / T\right)}
$$

As a result of it, expression for $\eta$ stated in (21) can also be expressed as

$$
\ln \eta=\ln \eta_{0}+\frac{D X(T)}{T \Delta S}
$$

where

$$
\left(X^{-1}(T)\right)=\frac{\frac{8}{3} \frac{T_{0}}{T}\left(1-\frac{T_{0}}{4 T}\right)}{\left(1+2 T_{0} / T\right)\left(1-T_{0} / 3 T\right)}
$$

is a weak temperature dependence function. In view of (20) and (23), one can say that, the expression for $\eta$ stated in (20) based on the free volume approach reduces to (23) which shows a relation between $\eta$ and $\Delta S$ and it is similar to the expression proposed by Adam and Gibbs (1965). The temperature dependence of the viscosity can be obtained by substituting the expression for $\Delta S$ as stated in (14) and it will be discussed in the next section.

\section{Viscosity of undercooled liquids based on configurational entropy}

The viscosity of the glass-forming liquids has also been studied in terms of the configurational entropy $\left(\Delta S_{c}\right)$ of liquids by Adam and Gibbs (1965) using the molecularkinetic theory and it can be expressed as

$$
\eta=A_{0} \exp \left(\frac{C_{0}}{T \Delta S_{\mathrm{c}}}\right)
$$

where constant $C_{0}$ is related to the potential energy hindering the cooperative rearrangement per monomer segment and the critical entropy and it is treated as a constant. $A_{0}$ is also a constant. The configurational entropy $\left(\Delta S_{\mathrm{c}}\right)$ is given by

$$
\Delta S_{\mathrm{c}}=\int\left[C_{\mathrm{p}} \text { (liquid) }-C_{\mathrm{p}} \text { (glass) }\right] \mathrm{d} \ln T .
$$

Often, the experimental value of $C_{p}$ (glass) is very close to that of the crystal and shows similar temperature dependence (Gee and Melia 1970; Wong and Angell 1976). 
Consequently, the expression for $\Delta S$ given in (10) or (14) can be used in place of $\Delta S_{\mathrm{c}}$ to obtain the temperature dependence of $\eta$. The resulting expression takes the form

$$
\ln \eta=\ln \eta_{0}^{\prime}+\frac{C\left(1+T_{0} / 3 T\right)}{\left(T-T_{0}\right)}
$$

where $C=2 / 3 C_{0} \delta^{2} e^{2(1-\delta)} / \Delta C_{\mathrm{p}}^{\mathrm{m}}$ and can be treated as a constant.

The expression for viscosity obtained above as stated in (27) can be compared with the Vogel-Fulcher equation stated in (2). At high temperatures i.e. $T \gg T_{0}$, the term $T_{0} / 3 T$ is small compared to unity and expression for $\eta$ in (27) reduces to the Vogel-Fulcher equation stated in (2) as

$$
\ln \eta=\ln \eta_{0}^{\prime}+\frac{C}{T-T_{0}}
$$

with $B_{2}=C$ and $A_{2}=\eta_{0}^{\prime}$. At low temperatures, i.e. near the glass transition temperature, $T_{0} / T \simeq T_{0} / T_{\mathrm{g}}$ is approximately $3 / 4$ and (27) yields

$$
\ln \eta=\ln \eta_{0}^{\prime}+\frac{C_{1}}{T-T_{0}}
$$

where $C_{1}=1.25 C$ and once again it reduces to Vogel-Fulcher equation with $B_{2}=1.25 C$ which is in justification of the fact that the Vogel-Fulcher equation was able to explain the experimental data of $\eta$ at low as well as high temperatures with the aid of different sets of constants $\left(A_{2}, B_{2}\right.$ and $T_{0}$ as stated in (2)). In view of these findings, it can be said that the constant $B_{2}$ in the Vogel-Fulcher expression should be temperature dependent to the extent

$$
B_{2}=C\left(1+T_{0} / 3 T\right) \text {. }
$$

At the same time, it is interesting to note that the expression for $\eta$ stated in (27) has less number of adjustable parameters than in the case of the Vogel-Fulcher equation. In the present case, $T_{0}$ is not an adjustable parameter and it can be calculated using thermodynamic parameters $\Delta C_{\mathrm{p}}^{\mathrm{m}}$ and $\Delta S_{\mathrm{m}}$ with the aid of (12) while $T_{0}$ is treated as an adjustable parameter in the Vogel-Fulcher expression. The expression for the temperature dependence of $\eta$ reported in the present work is much simpler than the expression reported by Dubey and Ramachandrarao (1992) using the expression for $\Delta S$ based on Taylor series expansion. The constants $\eta_{0}^{\prime}$ and $C$ can be estimated with the help of experimental data of $\eta$ at any two temperatures (say $T_{\mathrm{g}}$ and $T_{\mathrm{m}}$ ) and $T_{0}$ can be evaluated with the aid of $\Delta C_{\mathrm{p}}^{\mathrm{m}}$ and $\Delta S_{\mathrm{m}}$ and (12). Thus, $\eta$ of the glass-forming melts can be calculated in the temperature range $T_{\mathrm{g}}$ to $T_{\mathrm{m}}$ with the aid of $\Delta C_{\mathrm{p}}^{\mathrm{m}}, \Delta S_{\mathrm{m}}$ and experimentally measured $\eta$ at $T_{\mathrm{g}}$ and $T_{\mathrm{m}}$.

\section{Results and discussion}

To test the applicability of expressions for thermodynamic parameters $\Delta H$ and $\Delta S$ used in the present analysis, $\Delta H$ and $\Delta S$ have been calculated for $A_{0.77} \mathrm{Ge}_{0.136} \mathrm{Si}_{0.094}$, $\mathrm{B}_{2} \mathrm{O}_{3}$ and 2-methylpentane belonging to metallic, oxide and molecular glass-forming liquids respectively in the temperature range $T_{\mathrm{g}}$ to $T_{\mathrm{m}}$ using the materials parameters stated in table 1 and results obtained are shown in figure 1 . The experimental data of 
Table 1. The materials constants used to study the temperature dependence of $\Delta H, \Delta S$ and viscosity.

\begin{tabular}{lccc}
\hline Parameters & $\mathrm{Au}_{0}=\mathrm{Ge}_{0136} \mathrm{Si}_{0096}$ & $\mathrm{~B}_{2} \mathrm{O}_{3}$ & $2-\mathrm{mp}$ \\
\hdashline $\mathrm{a}(\mathrm{J} / \mathrm{mol} / \mathrm{K})$ & 32.5264 & $70 \cdot 8540$ & 146.871 \\
$\mathrm{~b}\left(\mathrm{~J} / \mathrm{mol} / \mathrm{K}^{2}\right) \times 10^{3}$ & -63.0822 & -73.4160 & $-798 \cdot 5$ \\
$\mathrm{c}\left(\mathrm{J} / \mathrm{mol} / \mathrm{K}^{3}\right) \times 10^{5}$ & 3.4141 & - & $\cdots$ \\
$\mathrm{d}(\mathrm{J} / \mathrm{mol}) \times 10^{-2}$ & $1 \cdot 3004$ & - & \\
$\mathrm{e}(\mathrm{JK} / \mathrm{mol}) \times 10^{-4}$ & $2 \cdot 5188$ & $141 \cdot 12$ & - \\
$\Delta H_{\mathrm{m}}(\mathrm{J} / \mathrm{mol})$ & 10627 & 22261 & 6260 \\
$\left.\Delta S_{\mathrm{m}} \mathrm{J} / \mathrm{mol} / \mathrm{K}\right)$ & 17.03 & $30 \cdot 79$ & $52 \cdot 36$ \\
$\Delta C_{\mathrm{p}}^{\mathrm{m}}(\mathrm{J} / \mathrm{mol} / \mathrm{K})$ & 6.74 & 20.47 & 51.41 \\
$T_{\mathrm{m}}(\mathrm{K})$ & 625 & 723 & 119.55 \\
$T_{\mathrm{g}}(\mathrm{K})$ & 294 & 550 & 79.5 \\
$T_{0}(\mathrm{~K})$ & $199 \cdot 4$ & 336 & $60 \cdot 82$ \\
\hline
\end{tabular}

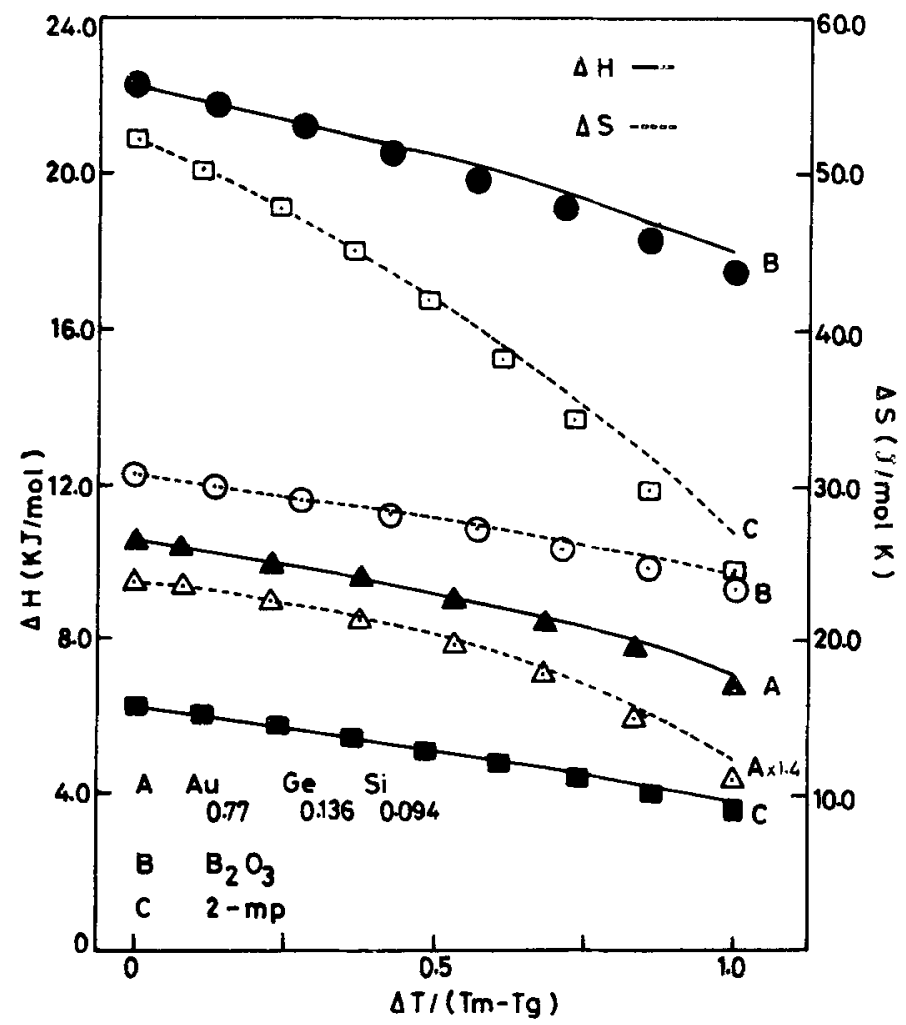

Figure 1. Variation of $\Delta H$ and $\Delta S$ with the reduced undercooling $\Delta T /\left(T_{\mathrm{m}}-T_{\mathrm{g}}\right)$. Curves $\mathrm{A}$, $\mathrm{B}$ and $\mathrm{C}$ correspond to glass-forming melts $\mathrm{Au}_{0}{ }_{77} \mathrm{Ge}_{0.136} \mathrm{Si}_{0.096}, \mathrm{~B}_{2} \mathrm{O}_{3}$ and 2-methylpentane respectively. Solid and dashed lines represent the calculated values of $\Delta H$ and $\Delta S$ respectively. Closed triangles, closed circles and closed squares represent the experimental values of $\Delta H$ for $\mathrm{Au}_{0.77} \mathrm{Ge}_{0136} \mathrm{Si}_{0096}, \mathrm{~B}_{2} \mathrm{O}_{3}$ and 2-mp respectively while open triangles, open circles and open squares denote the experimental data of $\Delta S$ of $\mathrm{Au}_{0.77} \mathrm{Ge}_{0.136} \mathrm{Si}_{0.096}, \mathrm{~B}_{2} \mathrm{O}_{3}$ and 2-mp respectively. For the sake of clarity $\Delta S$ of $A u_{077} \mathrm{Ge}_{0136} \mathrm{Si}_{0094}$ has been enlarged by a factor of 1.4 . 


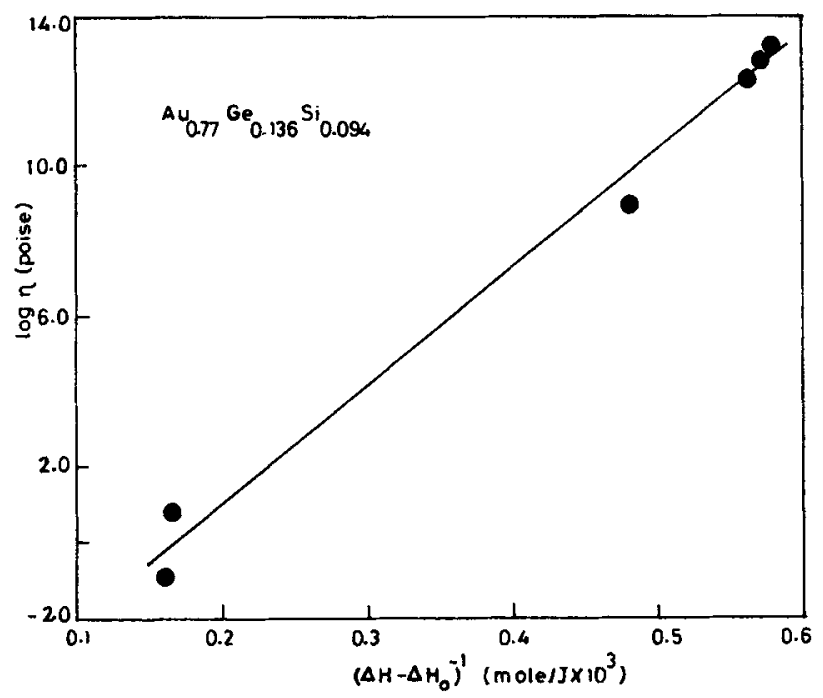

Figure 2. Variation of $\log \eta$ with $\left(\Delta H-\Delta H_{0}\right)^{-1}$ for the glass-forming melt $A u_{0.77} \mathrm{Ge}_{0.136} \mathrm{Si}_{0.096}$. Circles represent experimental data of $\eta$.

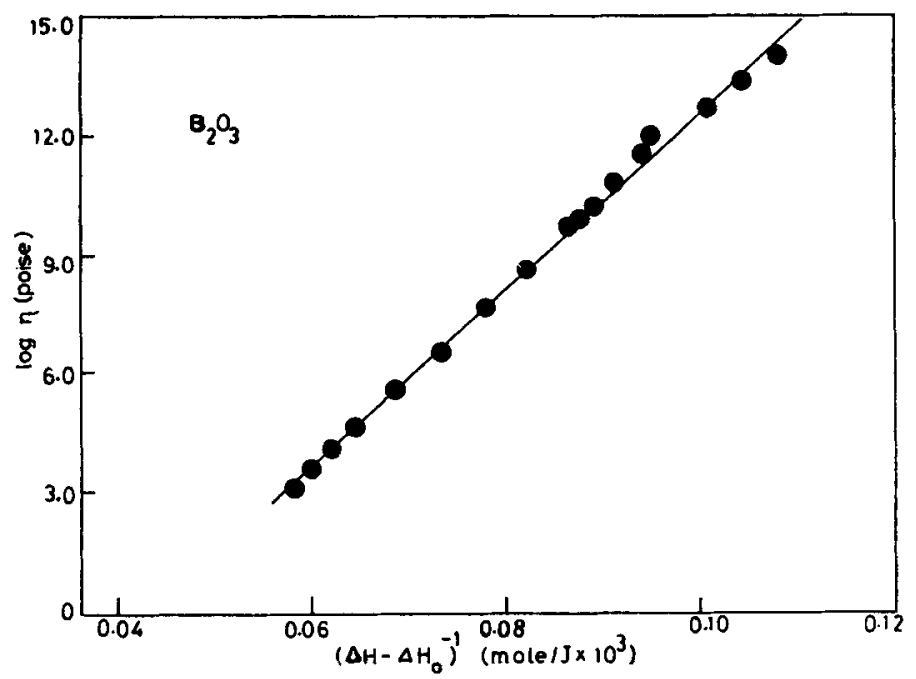

Figure 3. Variation of $\log \eta$ with $\left(\Delta H-\Delta H_{0}\right)^{-1}$ for the glass-forming melt $\mathrm{B}_{2} \mathrm{O}_{3}$. Circles correspond to experimental values of $\eta$.

$\Delta C_{\mathrm{p}}$ of these materials are available in literature (Thomas and Parks 1931; Douslin and Huffmann 1946; Greet and Turnbull 1967; Chen and Turnbull 1968; Angell and Rao 1972; Hultgren et al 1973) in the form of

$$
\Delta C_{\mathrm{p}}=a+b T=c T^{2}+d / T+e / T^{2}
$$

where $a, b, c, d$ and $e$ are the materials constants. With the help of figure 1 , it is very clear that the agreement between calculated and experimental values of $\Delta H$ and $\Delta S$ are quite good for all the three materials. 


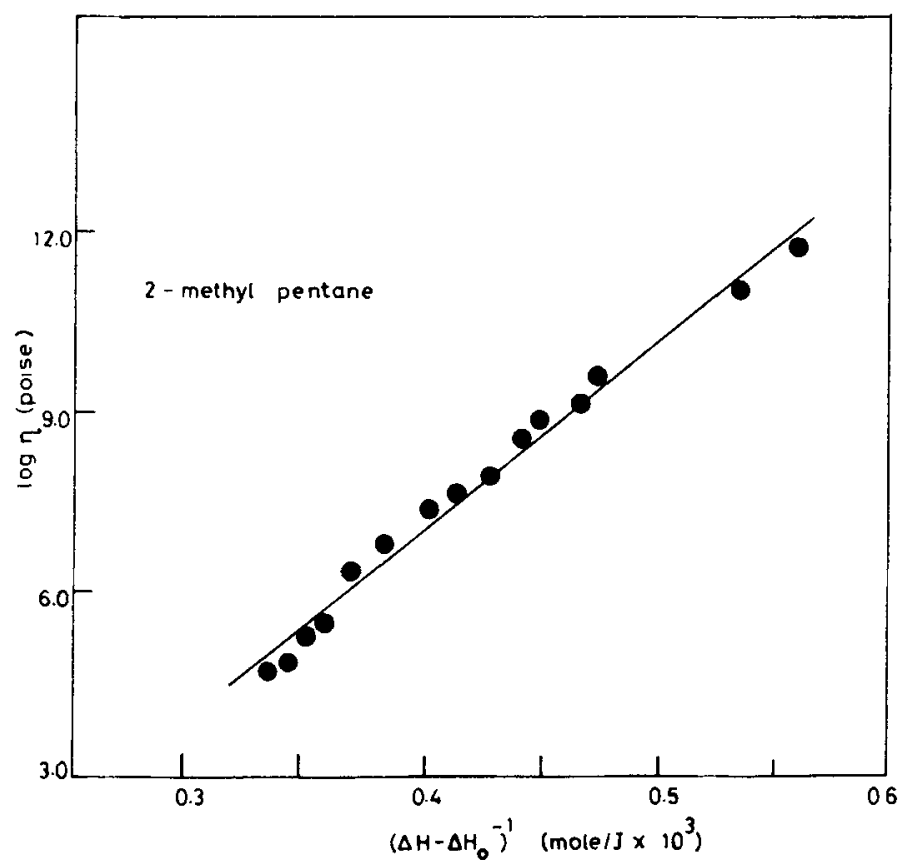

Figure 4. Variation of $\log \eta$ with $\left(\Delta H-\Delta H_{0}\right)^{-1}$ for the glass-forming melt 2-methylpentane. Circles denote the experimentally measured values of $\eta$.

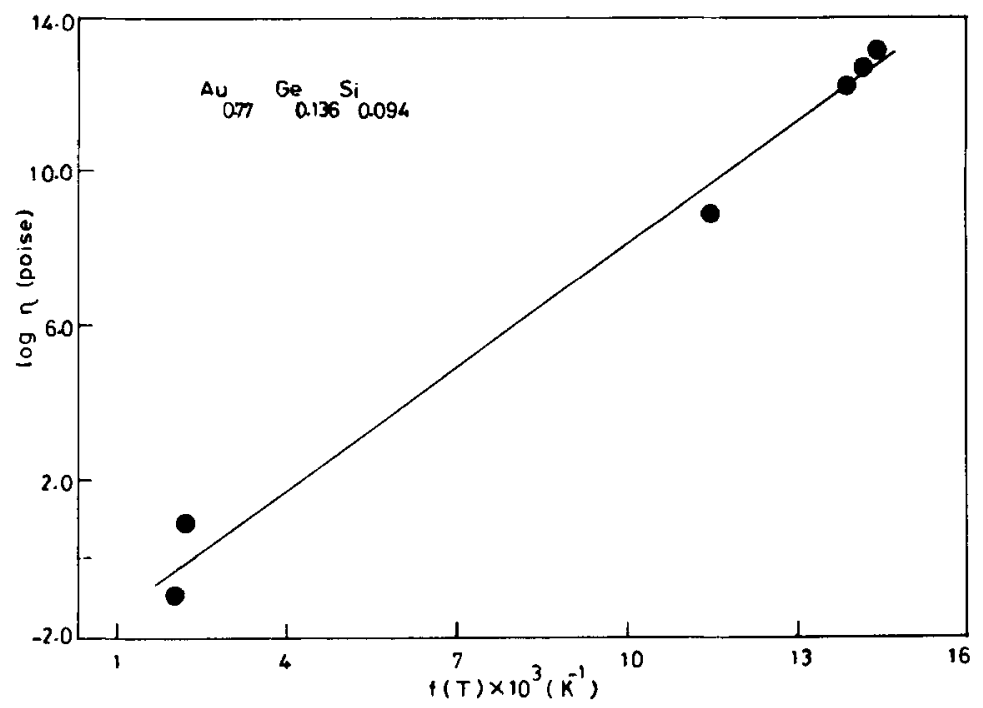

Figure 5. The temperature dependence of $\eta$ of the metallic melt $A u_{0.77} \mathrm{Ge}_{0.136} \mathrm{Si}_{0096}$ as predicted by (27). Circles represent the experimentally observed values of $\eta$.

To see the applicability of the expression for $\eta$ reported in (20), the viscosity of $\mathrm{Au}_{0.77} \mathrm{Ge}_{0.136} \mathrm{Si}_{0.096}, \mathrm{~B}_{2} \mathrm{O}_{3}$ and 2-methylpentane (2-mp) have been shown as a function of $\left(\Delta H-\Delta H_{0}\right)^{-1}$ in figures 2,3 and 4 respectively. The experimental values of the viscosity of the metallic glass $\mathrm{Au}_{0.77} \mathrm{Ge}_{0.136} \mathrm{Si}_{0.094}$ are rare. Chen and Turnbull (1968) 


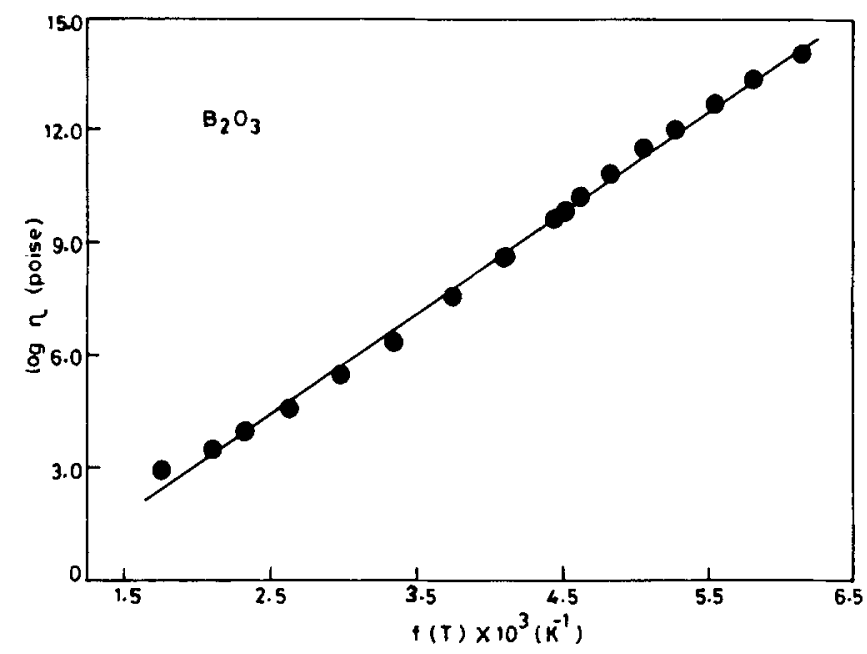

Figure 6. The temperature dependence of $\eta$ of the glass-forming melt $\mathrm{B}_{2} \mathrm{O}_{3}$ as obtained by (27). Circles stand for experimental data on $\eta$.

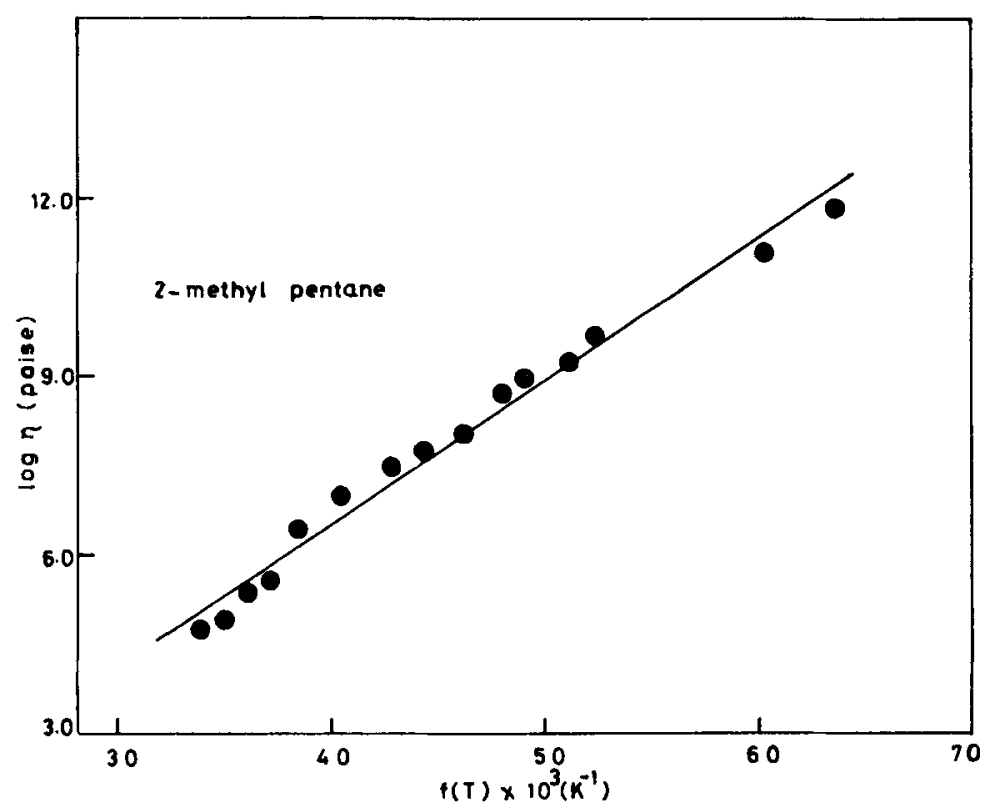

Figure 7. The temperature dependence of $\eta$ of the glass-forming liquid 2-methylpentane as reported in (27). Circles represent the experimentally measured values of $\eta$.

measured $\eta$ over a narrow temperature range (285-305 K) near $T_{\mathrm{g}}$ and Pok and Turnbull (1972) subsequently measured $\eta$ of the same at two temperatures 679 and $721 \mathrm{~K}$. The earlier workers (Pok and Turnbull 1972) tried to explain these data using the Vogel-Fulcher equation and they found that one set of constants $\left(A_{2}, B_{2}\right.$ and $T_{0}$ in (2)) can not adequately fit the data over the entire temperature range. The experimental data of $\eta$ for $\mathrm{B}_{2} \mathrm{O}_{3}$ and 2-mp are taken from the earlier report of Napolitano et al (1965) and Ling and Williard (1968) respectively. With the help of figures $2-4$, it can be seen 


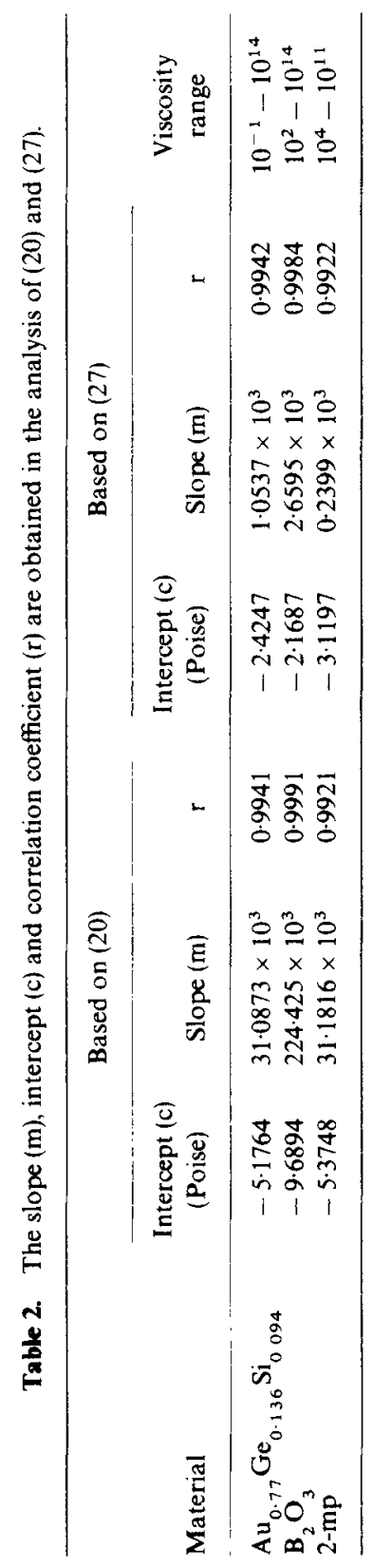


that the plot of $\log \eta$ against $\left(\Delta H-\Delta H_{0}\right)^{-1}$ is a straight line for all materials and correlation is also quite good. Thus, the viscosity of the glass-forming liquids can be estimated with a knowledge of enthalpy difference $\Delta H$ which can be calculated with the aid of $\Delta C_{\mathrm{p}}^{\mathrm{m}}, \Delta S_{\mathrm{m}}$ and $T_{\mathrm{m}}$. During the numerical analysis, it has also been noticed that the contribution of term $\left(\Delta G_{0}-\Delta G\right) / T \Delta S$ in (21) is small at low temperature i.e. around $T_{\mathrm{g}}$ as compared to its contribution at higher temperature i.e. near $T_{\mathrm{m}}$.

The temperature dependence of the viscosity has also been derived on the basis of the configurational entropy of liquids introducing the temperature dependence of $\Delta C_{p}$ and the expression obtained is reported in (27). To see the validity of this expression, the experimentally determined values of $\eta$ have been illustrated as a function of $f(T)=\left(1+T_{0} / 3 T\right) /\left(T-T_{0}\right)$ for $\mathrm{Au}_{0.77} \mathrm{Ge}_{0.136} \mathrm{Si}_{0.094}, \mathrm{~B}_{2} \mathrm{O}_{3}$ and 2-mp in figures 5, 6 and 7 respectively which show the plot of $\log \eta$ against $f(T)$ is a straight line for all three materials. The intercepts and slopes are stated in table 2 along with the correlation coefficient $r$ for the best fit straight line. With the help of figures 5-7, and the correlation coefficients stated in table 2 , it can be seen that a single set of constants $\left(\eta_{0}^{\prime}\right.$, $C$ and $T_{0}$ ) is able to fit the experimental data of the viscosity for all the three samples in the entire temperature range of study. In view of these results, one can say that the expression for $\eta$ reported in (27) is able to represent the temperature dependence of the viscosity correctly for metallic, oxide and molecular glass-forming liquids. It can also be said that the constant $B_{2}$ in the Vogel-Fulcher expression (2) should be taken as temperature dependent as stated in (30). A similar temperature dependence of viscosity can also be derived using (20) or (23) based on the free volume theory.

\section{Conclusion}

The expressions for the thermodynamic parameters reported by the authors have been used to discuss the viscous behaviour of metallic, oxide and molecular glass-forming liquids on the basis of the free volume theory as well as on the basis of configurational entropy model. The expression for $\eta$ reported in (20) based on the free volume theory is capable to explain the viscous behaviour of the glass-forming liquids with the help of single set of constants $\eta_{0}, D$ and $T_{0}$. The expression derived for the temperature dependence of viscosity stated in (27) is a general form of the Vogel-Fulcher equation and it does not involve any more adjustable parameters compared to the VogelFulcher equation. At the same time, it modifies the results in a significant fashion. The expression obtained is capable of reproducing the temperature dependence of viscosity with a single set of constants $\eta_{0}^{\prime}, C$ and $T_{0}$ over a wide range of viscosity $\left(10^{-1}\right.$ to $10^{14}$ poise) which suggests its great applicability. The viscous behaviour of the glass-forming melts can be studied with the aid of the thermodynamic parameters $\Delta C_{\mathrm{p}}^{\mathrm{m}}$ and $\Delta S_{\mathrm{m}}$ and these two parameters can be measured experimentally.

\section{Acknowledgements}

The authors are grateful to Prof. P Ramachandrarao, NML, Jamshedpur and Prof. S Lele, Department of Metallurgical Engineering, BHU Varanasi, for their interests in the present work. Part of work was carried out under the financial support by CSIR, New Delhi and the authors are thankful for that. 


\section{References}

Adam G and Gibbs J H 1965 J. Chem. Phys. 43139

Angell C A and Rao K J 1972 J. Chem. Phys. 57470

Bondi A 1954 J. Phys. Chem. 58929

Bueche F 1959 J. Chem. Phys. 30748

Chen H S and Turnbull D 1968 J. Chem. Phys. 482560

Cohen M H and Turnbull D 1959 J. Chem. Phys. 311164

Cohen M H and Grest G S 1979 Phys. Rev. B20 438

Davies H A 1976 Phys. Chem. Glasses 17159

Doolittle A K 1951 J. Appl. Phys. 22471

Dubey K S and Ramachandrarao P 1984 Acta Metall. 3291

Dubey K S and Ramachandrarao P 1992 Bull. Mater. Sci. 15111

Douslin D R and Huffmann H M 1946 J. Am. Chem. Soc. 681704

Dunn J S 1926 Trans. Faraday Soc. 22401

Flory P J 1953 Principles of polymer chemistry (New York: Cornell Univ. Press)

Frankel J 1955 Kinetic theory of liquids (New York: Dover)

Fulcher G S 1925 J. Am. Ceram. Soc. 773701

Gee D R and Melia T P 1970 Makromol. Chem. 132195

Glasstone S, Laidler K J and Eyring H 1941 Theory of rate processes (New York: McGraw-Hill) Chap. 9

Greet R J and Turnbull D 1967 J. Chem. Phys. 472185

Hirai N and Erying H 1958 J. Appl. Phys. 29810

Hirai N and Erying H 1959 J. Polym. Sci. 3751

Hultgren R, Desai P D, Hawkins D J, Gleiser M, Kelly K K and Wagman D D 1973 Selected values of thermodynamic properties of the elements (Ohio: Am. Soc. Metals)

Kauzmann W 1948 Chem. Rev. 43219

Ling A C and Williard J E 1968 J. Phys. Chem. 721918

Macedo P B and Litovitz T A 1965 J. Chem. Phys. 42245

Mishra R K and Dubey K S 1996 Bull. Mater. Sci. 19357

Napolitano A, Macedo P B and Hawkins E G 1965 J. Am. Ceram. Soc. 48613

Pok D E and Turnbull D 1972 Acta Metall. 20493

Ramachandrarao P, Cantor B and Cahn R W 1977 J. Non-Cryst. Solids 24109

Raman C V 1923 Nature 111532

Ree T S, Ree T and Erying H 1962 Proc. Nat. Acad. Sci. USA 48501

Sanchez I C 1974 J. Appl. Phys. 454204

Thomas S B and Parks G S 1931 J. Phys. Chem. 472091

Turnbull D and Cohen M H 1961 J. Chem. Phys. 34120

Turnbull D and Cohen M H 1970 J. Chem. Phys. 523038

Uhlmann D R 1972 J. Non-Cryst. Solids 7337

Vogel H 1921 Phys. Z. 22645

Wong J and Angell C A 1976 Glass: Structure by spectroscopy (New York: Marcel Dekker) pp 1-51 